\title{
Stroke-Like Migraine Attacks After Radiation Therapy Syndrome
}

\author{
Daniel April, MD, ${ }^{1}$ Neil Lall, MD, ${ }^{2}$ Andrew Steven, MD $^{1}$ \\ ${ }^{1}$ Department of Radiology, Ochsner Clinic Foundation, New Orleans, LA ²Department of Radiology, Emory University School of Medicine, \\ Atlanta, GA
}

\section{INTRODUCTION}

Stroke-like migraine attacks after radiation therapy (SMART) syndrome is a delayed complication of brain irradiation. ${ }^{1}$ Symptoms are varied but typically consist of recurrent migraines with subsequent stroke-like symptoms and seizures. ${ }^{2-5}$ The associated imaging findings are unusual but can be quite characteristic on magnetic resonance imaging (MRI) and include unilateral increased T2 signal with associated gyral thickening and enhancement in the temporal, parietal, and occipital lobes. ${ }^{1}$ The pathogenesis is not as well understood as with other complications of radiation, such as leukoencephalopathy and radiation necrosis, but familiarity with this rare clinicoradiologic syndrome is important. We describe the case of a patient with a typical clinical presentation and imaging findings of SMART syndrome.

\section{HISTORY AND CASE REPORT}

A 58-year-old female presented to the emergency department (ED) with a 2-day history of severe headache, rightsided hemiparesis, involuntary eye blinking, slurred speech, and confusion. Her medical history included hypertension, hyperlipidemia, type 2 diabetes mellitus, and transient ischemic attacks. Additionally, she had a history of stage IV breast cancer with an isolated left frontal lobe brain metastasis. She had undergone surgical excision and brain irradiation therapy 8 years prior to presentation. The patient had visited the ED several times during the prior 3 years with syncopal episodes and confusion. Computed tomography (CT) and MRI of the brain performed at these visits demonstrated stable postoperative and radiation changes in the left frontal lobe at the site of the patient's known metastatic disease. Two electroencephalograms (EEGs) performed at these visits demonstrated generalized slowing in the left frontal lobe without epileptiform activity. These episodes were treated as absence seizures, and she was prescribed levetiracetam (Keppra) $1 \mathrm{~g}$ twice daily for management.

At this most recent ED visit, initial CT and MRI of the brain revealed stable posttreatment changes, as well as mild cerebral atrophy and chronic ischemic microvascular changes, but no acute abnormalities. The patient was admitted to the hospital, and initial complete blood count (CBC), blood culture, and paraneoplastic panel were negative. Initial lumbar puncture (LP) showed elevated white blood cells, and meningitis was suspected. On day 1 of admission, empiric antiviral treatment and antibiotics were started: intravenous acyclovir $640 \mathrm{mg} 3$ times daily for 9 days, fluconazole $400 \mathrm{mg}$ once daily for 5 days, and piperacillin-tazobactam $4.5 \mathrm{~g}$ once daily for 3 days. Because of the concern for seizure, $1,000 \mathrm{mg}$ of levetiracetam twice daily was initiated. Initial EEG revealed bilateral hemispheric slowing without focal epileptiform activity. Cerebrospinal fluid (CSF) cultures showed no growth, and repeat LP on hospital day 6 was negative. The patient had continued episodes of confusion, and because of concern for persistent seizure activity, on hospital day 7 , her levetiracetam dose was increased to $1,500 \mathrm{mg}$ twice daily, and lacosamide $100 \mathrm{mg}$ was added on hospital day 8. On hospital day 9, the patient had a witnessed seizure with eye blinking and right-sided facial twitching that was stopped with lorazepam. Repeat MRI exhibited new mild cortical expansion and enhancement, with increased signal on T2-weighted images and diffusion restriction involving the posterior left cerebral hemisphere (Figure 1). Her lacosamide dose was increased to $200 \mathrm{mg}$ twice daily, and she was transferred to the intensive care unit for further monitoring.

On hospital day 11, the patient was found unresponsive; she was intubated and transferred for a higher level of care and continuous EEG monitoring. She was extubated 13 days later, and repeat MRI showed persistent but decreased enhancement with slightly decreased hyperintensity in the posterior left cerebral cortex and persistence of diffusion restriction (Figure 2). Given the imaging appearance, evolution of imaging findings, and the patient's clinical history, a diagnosis of SMART syndrome was suspected.

The patient continued to improve slowly, but she had persistent altered mentation, aphasia, and difficulty performing activities of daily living and required percutaneous endoscopic gastrostomy (PEG) tube placement. On hospital day 20 , she was switched to and maintained on levetiracetam $750 \mathrm{mg}$ twice daily.

Thirty-six days after hospital admission, the patient was transferred to a rehabilitation facility where she stayed for 1 month. Upon admission to the rehabilitation facility, she was unable to stand or walk on her own, had receptive aphasia, and had significant swallowing and speech impairment. She made significant improvement during her rehabilitation stay, regaining her ability to walk short distances and to communicate in simple sentences. She was advanced to a soft diet and discharged home with continued weekly rehabilitation and in-home assistance with performing daily tasks. Discharge antiepileptic medication included only levetiracetam 1,000 mg twice daily. One month after discharge from the rehabilitation facility, her PEG tube was removed. During 


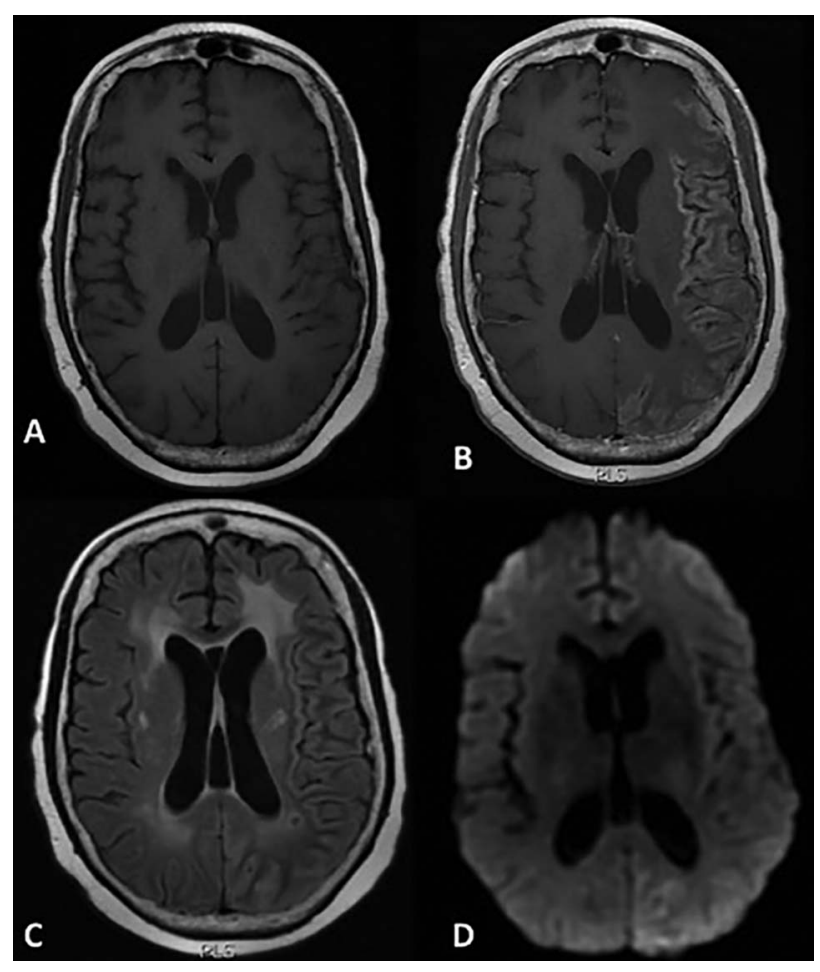

Figure 1. Magnetic resonance imaging on hospitalization day 9. Axial T1-weighted images through the level of the cerebral hemispheres before (A) and after (B) the administration of gadolinium contrast demonstrate diffuse gyral enhancement throughout much of the left cerebral hemisphere with some sparing of the frontal lobe, with corresponding mildly hyperintense signal on a T2/fluid attenuated inversion recovery image $(C)$ and diffusion restriction on diffusion weighted imaging (D).

several follow-up outpatient visits, she was noted to have ongoing upper extremity weakness and difficulty with her gait, as well as decreased coordination.

On 5-month follow-up MRI, the cortical enhancement and diffusion restriction had almost completely resolved with minimal residual laminar necrosis (Figure 3).

\section{RADIOGRAPHIC APPEARANCE AND TREATMENT}

$M R I$ is the mainstay of radiologic diagnosis for SMART syndrome as CT typically will not illustrate any abnormalities. Characteristic MRI findings include unilateral thick gyriform cortical enhancement and corresponding T2/fluidattenuated inversion recovery (FLAIR) hyperintensity. ${ }^{1,6}$ The most commonly affected areas include the occipital, parietal, and posterior frontal lobes. Subcortical and deep white matter is typically spared. Findings also do not tend to involve the deep gray nuclei or posterior fossa structures. Imaging findings are often transient and reversible. Although enhancement can last up to 12 weeks, it typically lasts 2 to 5 weeks. ${ }^{1,3,4}$ Diffusion restriction is often present but may be absent in the region of cortical abnormalities. ${ }^{1}$ In a case series by Black et al, 3 of 11 patients demonstrated diffusion restriction, but the authors hypothesized that the diffusion restriction could be attributed to superimposed infarcts. ${ }^{1}$ Biopsy is not typically indicated, as histopathologic findings

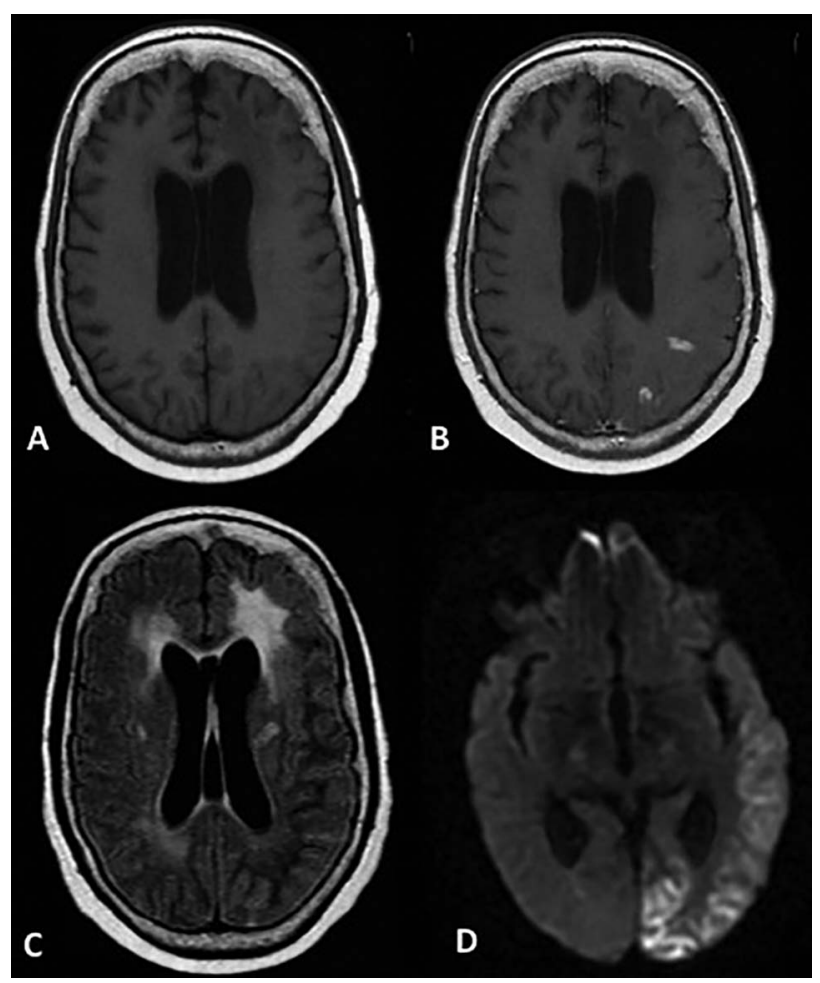

Figure 2. Follow-up magnetic resonance imaging 1 week later. Axial T1-weighted images through the level of the cerebral hemispheres before (A) and after (B) the administration of gadolinium contrast demonstrate few remaining foci of cortical enhancement involving the left posterior parietal and occipital lobes, with corresponding mild residual T2/fluid attenuated inversion recovery signal along the left occipital lobe (C) and persistent cortical restricted diffusion involving the left posterior parietal and occipital lobes on diffusion weighted imaging (D).

are nonspecific and SMART syndrome is a clinicoradiologic diagnosis. $^{7}$

There is no clear consensus regarding treatment, but therapy is usually targeted toward symptom control. In the acute setting, immediate control of seizures is important and can be accomplished with anticonvulsive therapy such as intravenous levetiracetam or fosphenytoin. Abend et al suggested that early administration of levetiracetam is effective in the treatment of status epilepticus in patients diagnosed with SMART syndrome. ${ }^{8}$

\section{DISCUSSION}

SMART syndrome is a delayed complication of both whole brain irradiation and selective radiosurgery. The onset of symptoms varies widely from 1 to 37 years after initial radiation therapy, with an average 9.5 years reported by Black et al and 20 years reported by Maloney et al. ${ }^{1,9}$ SMART syndrome has been described in both children and adults and is typically associated with radiation doses of 50 Gy or more, although it can occur with lower doses. ${ }^{10-12}$ Acute symptoms consist primarily of headaches, often with a migraine-like aura. Subsequently, patients often develop confusion, seizures, and stroke-like symptoms that may include motor deficits, paresthesia, aphasia, and visual 


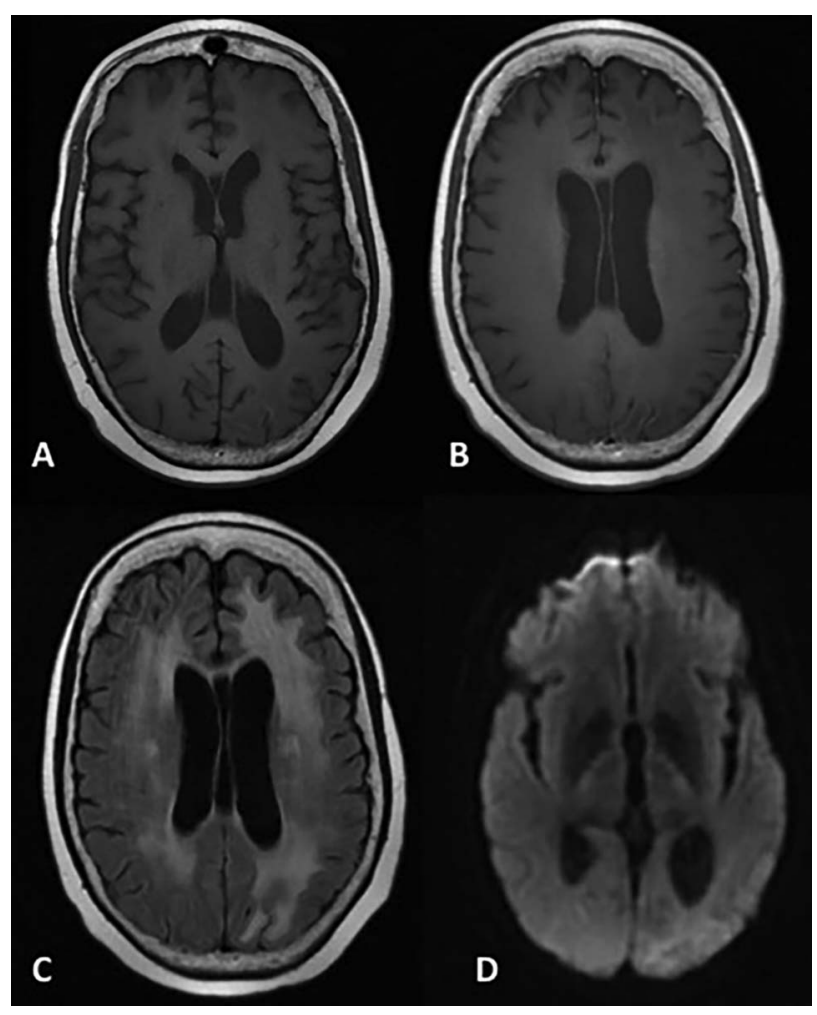

Figure 3. Magnetic resonance imaging at 5-month followup. Axial T1-weighted images before $(A)$ and after $(B)$ the administration of gadolinium contrast demonstrate minimal cortical intrinsic T1 shortening along the left posterior parietal and occipital cortex consistent with laminar necrosis but complete resolution of the postcontrast cortical enhancement. T2/fluid attenuated inversion recovery image demonstrates resolution of the posterior cerebral cortical signal abnormality but new left occipital subcortical hyperintensity, indicative of gliosis and encephalomalacia (C). Diffusion weighted imaging shows resolution of diffusion restriction (D).

disturbances. Symptoms last from several hours to several weeks. Some patients experience full recovery, while others have recurrent transient episodes and incomplete recovery with long-term residual neurologic deficits. ${ }^{9,13}$

Black et al proposed a revision of the Bartleson diagnostic criteria that includes several defining features of the syndrome and MRI findings. ${ }^{2}$ The criteria include a remote history of external beam brain irradiation without residual or recurrent disease with symptoms that are referable to a unilateral cortical region. These manifestations include hemisensory defects, hemiparesis, aphasia, seizures, headaches, and confusion. MRI findings consist of diffuse, transient cortical enhancement that often spares the white matter and cannot be attributable to another disorder.

The pathophysiology of SMART syndrome is not well understood. Radiation is a defining precursor and a known cause of delayed neurotoxicity that can result in leukoencephalopathy and radiation necrosis. ${ }^{14}$ Farid et al suggested that neuronal dysfunction plays a role in SMART syndrome. ${ }^{12}$ Impairment of the trigeminovascular system and ion channels leads to disruption of the blood-brain barrier and sub- sequent lowered threshold for spreading of cortical depression, a process involved in the pathophysiology of migraine headaches. Black et al proposed that endothelial dysfunction may lead to disruption of the blood-brain barrier with subsequent marked cortical enhancement. ${ }^{1}$

Associated histopathologic findings include gliosis, perivascular cell infiltrates, and inflammation. ${ }^{7}$ Endothelial cell damage and vascular endothelial growth factor upregulation can be seen with smooth muscle proliferation and fibrinoid necrosis of the vessel wall. ${ }^{15-17}$ However, which, if any, of these factors is responsible for the clinical and radiologic findings is unclear, as case reports have reported no identifiable histopathologic abnormality. Shuper et al reported the case of a patient whose autopsy 1.5 years after the beginning of migraine headache episodes demonstrated no vascular damage. ${ }^{6}$ Black et al reported the case of a patient's brain biopsy that demonstrated nonspecific gliosis without inflammation or other histopathology related to SMART syndrome.

SMART syndrome is typically reversible. In the Rigamonti et al review of the literature, $83 \%$ of patients (30/36) were reported to have had a complete recovery. ${ }^{18}$ Di Stefano et al reported full recovery in $85 \%(22 / 26)$ of patients, while $15 \%$ were left with permanent symptoms. ${ }^{19}$ Black et al reported that $45 \%(5 / 11)$ of patients in their case series had longterm symptoms, including cognitive impairment, hemiparesis, and dysphasia. ${ }^{1}$ Permanent imaging findings of cortical laminar necrosis were present in $27 \%$ of patients.

\section{DIFFERENTIAL DIAGNOSIS}

The initial consideration when presented with enhancing lesions in a symptomatic patient with a history of treated malignancy is a recurrent neoplastic process. However, SMART syndrome can be distinguished by the characteristic gyriform cortical enhancement vs the solid or nodular pattern often seen in metastatic disease. CSF dissemination of malignancy can mimic the gyriform pattern of SMART syndrome, but the enhancement will be centered in the leptomeninges instead of the cortex.

Seizure-related signal changes in the setting of status epilepticus have some of the same imaging findings as SMART syndrome, including abnormal cortical and subcortical white matter T2/FLAIR hyperintensity and variable gyral or leptomeningeal enhancement. ${ }^{4}$ Similarly, these signal changes are not typically confined to vascular territories. In seizures, the pattern of T2/FLAIR hyperintensity often prominently involves the hippocampus, corpus callosum splenium, and thalamus, which is not typical for SMART syndrome.

Subacute infarcts often exhibit gyriform enhancement. However, findings should remain confined to a vascular territory, and the clinical scenario will differ. Our patient presented with recent-onset slurred speech and right-sided hemiparesis, initially concerning for an acute ischemic infarct. This diagnosis was ruled out when the initial MRI did not demonstrate diffusion restriction or additional findings consistent with ischemia. Additionally, in early ischemia, loss of the gray-white differentiation and edema is typical and was not demonstrated in our patient.

Infectious processes, such as encephalitis or meningitis, can have imaging characteristics similar to SMART syndrome. In meningitis, leptomeningeal T2/FLAIR hyperinten- 
sity and enhancement are often seen, as well as an exudative process in the sulci and basilar cisterns that often results in hydrocephalus. Typical imaging findings in several encephalitides are T2/FLAIR hyperintensity in the limbic system, often bilaterally. Cortical swelling with loss of gray-white differentiation can also occur, often in the medial temporal lobes and insula with sparing of the basal ganglia. Subacute hemorrhage can occur within edematous areas. These findings do not occur in SMART syndrome.

As in SMART syndrome, posterior reversible encephalopathy syndrome (PRES) demonstrates endothelial damage and vasculopathy with increased parietocortical FLAIR signal because of vasogenic edema. In PRES, however, imaging findings are typically bilateral, and enhancement is only seen in a minority of cases.

\section{CONCLUSION}

In a patient with stroke-like symptoms, migraines, and seizures; with MRI findings of gyriform enhancement; and with a history of prior brain irradiation, SMART syndrome should be the leading diagnosis in the differential. Appropriate clinical workup is essential to exclude other potential etiologies. Familiarity with the characteristic findings of SMART syndrome and the subtle distinguishing imaging features can prevent unnecessary and invasive testing.

\section{ACKNOWLEDGMENTS}

The authors have no financial or proprietary interest in the subject matter of this article.

\section{REFERENCES}

1. Black DF, Morris JM, Lindell EP, et al. Stroke-like migraine attacks after radiation therapy (SMART) syndrome is not always completely reversible: a case series. AJNR Am J Neuroradiol. 2013 Dec;34(12):2298-2303. doi: 10.3174/ajnr.A3602.

2. Black DF, Bartleson JD, Bell ML, Lachance DH. SMART: stroke-like migraine attacks after radiation therapy. Cephalalgia. 2006 Sep;26(9):1137-1142. doi: 10.1111/j.1468-2982.2006.01184.x.

3. Pruitt A, Dalmau J, Detre J, Alavi A, Rosenfeld MR. Episodic neurologic dysfunction with migraine and reversible imaging findings after radiation. Neurology. 2006 Aug 22;67(4):676-678. doi: 10.1212/01.wnl.0000228862.76269.62.

4. Partap S, Walker M, Longstreth WT Jr, Spence AM. Prolonged but reversible migraine-like episodes long after cranial irradiation. Neurology. 2006 Apr 11;66(7):1105-1107. doi: 10.1212/01.wnl.0000204178.65221.b8.

5. Cordato DJ, Brimage P, Masters LT, Butler P. Post-cranial irradiation syndrome with migraine-like headaches, prolonged and reversible neurological deficits and seizures. J Clin Neurosci. 2006 Jun;13(5):586-590. doi: 10.1016/j.jocn.2005.04.026.

6. Shuper A, Packer RJ, Vezina LG, Nicholson HS, Lafond D. Complicated migraine-like episodes in children following cranial irradiation and chemotherapy. Neurology. 1995 Oct;45(10):1837-1840. doi: 10.1212/WNL.45.10.1837.

7. Brown WR, Thore CR, Moody DM, Robbins ME, Wheeler KT. Vascular damage after fractionated whole-brain irradiation in rats. Radiat Res. 2005 Nov; 164(5):662-668. doi: $10.1667 / \mathrm{rr} 3453.1$.

8. Abend NS, Florance N, Finkel RS, Licht DJ, Dlugos DJ. Intravenous levetiracetam terminates refractory focal status epilepticus. Neurocrit Care. 2009;10(1):83-86. doi: 10.1007/s12028-007-9044-9.

9. Maloney PR, Rabinstein AA, Daniels DJ, Link MJ. Surgically induced SMART syndrome: case report and review of the literature. World Neurosurg. 2014 Jul-Aug;82(1-2):240.e7-e12. doi: 10.1016/j.wneu.2013.01.028.

10. Bartleson JD, Krecke KN, O'Neill BP, Brown PD. Reversible, strokelike migraine attacks in patients with previous radiation therapy. Neuro Oncol. 2003 Apr;5(2):121-127. doi: 10.1093/neuonc/5.2.121.

11. Kerklaan JP, Lycklama á Nijeholt GJ, Wiggenraad RG, Berghuis B, Postma TJ, Taphoorn MJ. SMART syndrome: a late reversible complication after radiation therapy for brain tumours. $J$ Neurol. 2011 Jun;258(6):1098-1104. doi: 10.1007/s00415-010-5892-x.

12. Farid K, Meissner WG, Samier-Foubert A, et al. Normal cerebrovascular reactivity in stroke-like migraine attacks after radiation therapy syndrome. Clin Nucl Med. 2010 Aug;35(8):583-585. doi: 10.1097/RLU.0b013e3181e4db6f.

13. Bradshaw J, Chen L, Saling M, Fitt G, Hughes A, Dowd A. Neurocognitive recovery in SMART syndrome: a case report. Cephalalgia. 2011 Feb;31(3):372-376. doi: $10.1177 / 0333102410388436$.

14. Rahmathulla G, Marko NF, Weil RJ. Cerebral radiation necrosis: a review of the pathobiology, diagnosis and management considerations. J Clin Neurosci. 2013 Apr;20(4):485-502. doi: 10.1016/j.jocn.2012.09.011.

15. Peña LA, Fuks Z, Kolesnick RN. Radiation-induced apoptosis of endothelial cells in the murine central nervous system: protection by fibroblast growth factor and sphingomyelinase deficiency. Cancer Res. 2000 Jan 15;60(2):321-327.

16. Nordal RA, Nagy A, Pintilie M, Wong CS. Hypoxia and hypoxia-inducible factor- 1 target genes in central nervous system radiation injury: a role for vascular endothelial growth factor. Clin Cancer Res. 2004 May 15;10(10):3342-3353. doi: 10.1158/1078-0432.CCR-03-0426.

17. Remler MP, Marcussen WH, Tiller-Borsich J. The late effects of radiation on the blood brain barrier. Int J Radiat Oncol Biol Phys. 1986 Nov;12(11):1965-1969. doi: 10.1016/0360-3016(86)90133-1.

18. Rigamonti A, Lauria G, Mantero V, Filizzolo M, Salmaggi A. SMART (stroke-like migraine attack after radiation therapy) syndrome: a case report with review of the literature. Neurol Sci. 2016 Jan;37(1):157-161. doi: 10.1007/s10072-015-2396-6.

19. Di Stefano AL, Berzero G, Ducray F, et al. Stroke-like events after brain radiotherapy: a large series with long-term follow-up. Eur J Neurol. 2019 Apr;26(4):639-650. doi: 10.1111/ene.13870.

This article meets the Accreditation Council for Graduate Medical Education and the American Board of Medical Specialties Maintenance of Certification competencies for Patient Care and Medical Knowledge.

(C)2020 by the author(s); licensee Ochsner Journal, Ochsner Clinic Foundation, New Orleans, LA. This article is an open (c) access article distributed under the terms and conditions of the Creative Commons Attribution (CC BY) license (creativecommons.org/licenses/by/4.0/legalcode) that permits unrestricted use, distribution, and reproduction in any medium, provided the original author(s) and source are credited. 